\title{
GROUPING OF RUNNERS DURING MARATHON COMPETITION
}

K. YAMAJI, PhD and R. J. SHEPHARD, MD, PhD

Laboratory for Exercise Physiology, Faculty of Education, Toyama University, Toyama, Japan and School of Physical and Health Education, University of Toronto

\section{ABSTRACT}

Marathon runners tend to form clusters during competition, possibly as a means of improving their performance. Studies of international competitors in Fukuoka and Tokyo races have shown a density of 0.44 to 0.80 runners. $\mathrm{m}^{2}$ among those maintaining a pace of 4.8-5.6 m.s ${ }^{-1}$, clustering being most marked among elite performers. Some $95 \%$ of clustered runners maintained a minimum distance of 0.5-1.5 m from other competitors; moreover, such competitors avoided occupying an angle of \pm 15 degrees either before or behind other runners. The phenomenon merits closer study by those interested in the bettering of marathon performance.

\section{INTRODUCTION}

Today's marathon races have been called "survival events". Typically, the top contestants form a cluster immediately after the start of the race and victory goes to the survivor among this elite group. Individual runners apparently adjust their pace quite finely so that they can sustain this clustering.

In order to study the phenomenon in detail, we mounted a video-camera on a high building at two points along the course of the 1985 Fukuoka International Marathon Race (at 9.8 and $20.0 \mathrm{~km})$, and at three points along the 1986 Tokyo International Marathon Race (at 4.8, 16.0 and $24.5 \mathrm{~km}$ ).

\section{METHODS}

Although the Fukuoka and Tokyo races attracted a fairly small number of competitors (137 and 68 respectively), they were international events with a fairly high level of performance, as can be judged from the pace at the $9.8 \mathrm{~km}$ mark in the Fukuoka race $\left(4.8\right.$ to $\left.5.6 \mathrm{~m} . \mathrm{s}^{-1}\right)$.
The video records were obtained as subjects ran away from the cameras. The inter-personal distance and angle were estimated by standard geometric principles, correcting for the height of the camera at each of the five selected sites and the distance of individual runners from the building where the camera was mounted. Distances between groups and between individual runners were calculated from the passing time of each competitor. For example, at the $9.8 \mathrm{~km}$ point in Fukuoka, the majority of competitors had grouped themselves into eight clusters (Fig. 1). Where there were more than 6 runners in a cluster the density of the group was also calculated (runners. ${ }^{2}$ ), based on the distance from the first to the last member of the cluster. A rectangle was created enclosing all members of the cluster, and this was arbitrarily divided into two segments (high density and low density); the mean density was then calculated as the average of densities for the two segments. Where the interpersonal distance was more than five metres, a competitor was considered as running alone.

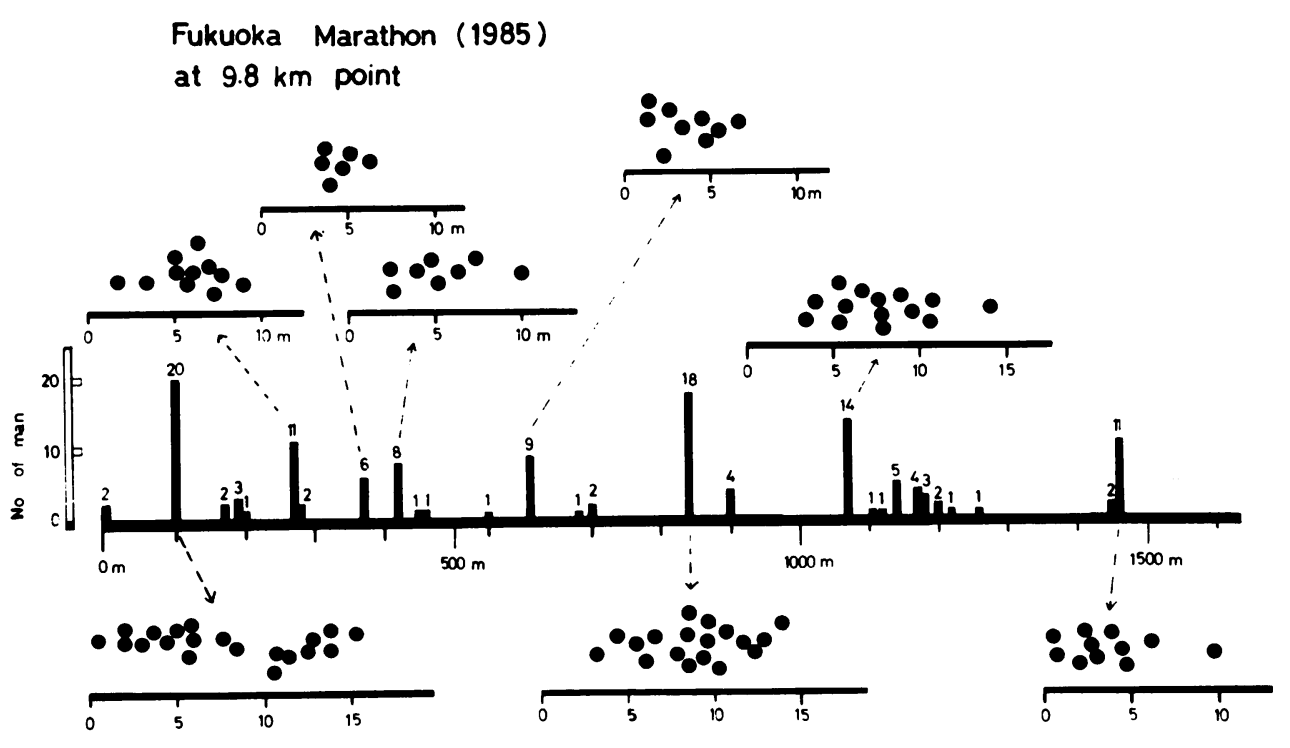

Fig. 1: Clustering of runners at the $9.8 \mathrm{~km}$ point in the Fukuoka Marathon, as estimated from passing times on a video record. The distribution of competitors within eight clusters is illustrated.

Address for correspondence:

Professor Roy J. Shephard, Director School of Physical \& Health Education 320 Huron Street

Toronto,

Ontario M5S $1 \mathrm{~A} 1$

Canada

\section{RESULTS}

The density of the clusters increased with running speed, from a value of 0.44 runners. $m^{2}$ at a pace of $4.8 \mathrm{~m}$.s, to 0.80 runners. $\mathrm{m}^{2}$ at $5.6 \mathrm{~m} . \mathrm{s}$ (Fig. 2). For $95 \%$ of the clustered runners, the shortest interpersonal distance was in the range 0.5-1.5 $\mathrm{m}$; frequency distribution curves for distances to the first, second, and third nearest rivals are shown in Fig. 3. 


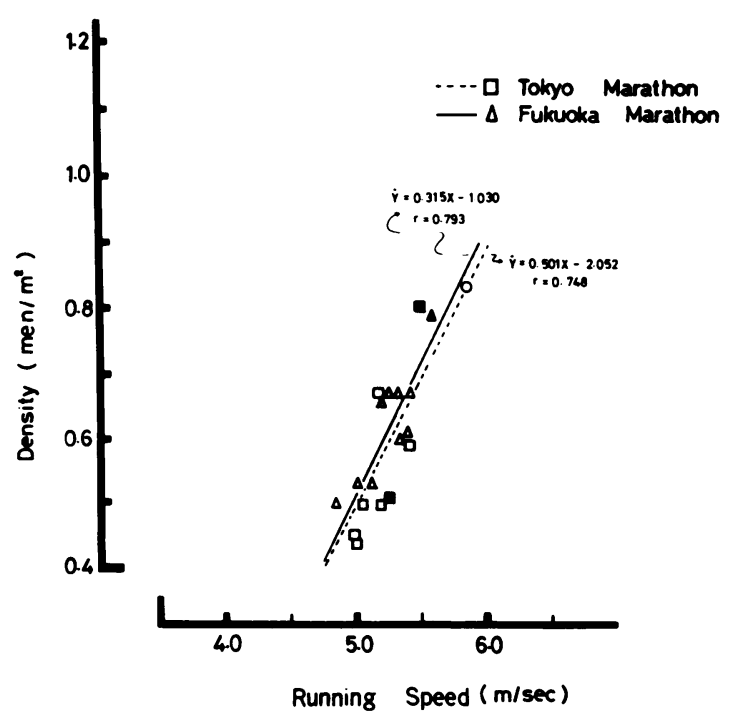

Fig. 2: The relationship between the density of competitor clusters and running speed. Data for the Tokyo and Fukuoka marathon races, with least squares regressions.

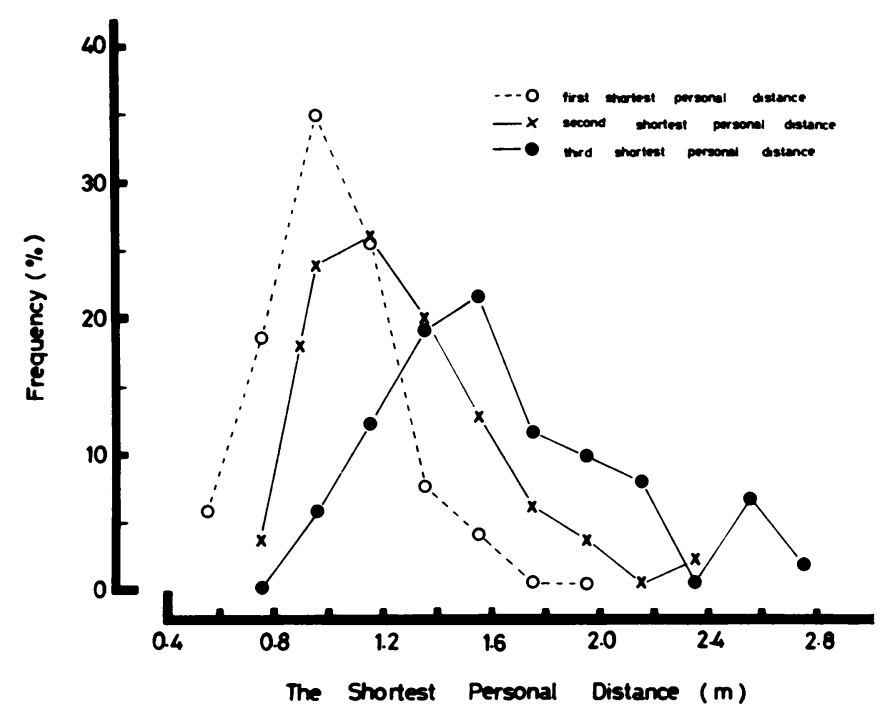

Fig. 3: Frequency distribution curves of inter-personal distances separating a competitor from the first, second and third closest rivals.

The angle separating a competitor from his nearest rivals is illustrated in Fig. 4; 95\% of other competitors avoided occupying the angle $\pm 15^{\circ}$ ahead and behind a given runner. This was probably partly to avoid collision if the front runner slowed his pace and partly because it was difficult to see the track when another person was directly ahead.

\section{DISCUSSION}

Our data support the view that marathon runners separate themselves from their nearest rivals by a relatively constant distance and angle throughout a race and further show that a clustering of competitors occurs at a variety of running speeds.

The anticipated tactical advantages from the observed grouping behaviour include (1) a reduction of air resistance, (2) an upsurge and maintenance of competitive spirit, (3) resolution of uncertainties regarding an appropriate running pace at various points in the race, with a consequent maximisation of mechanical efficiency of

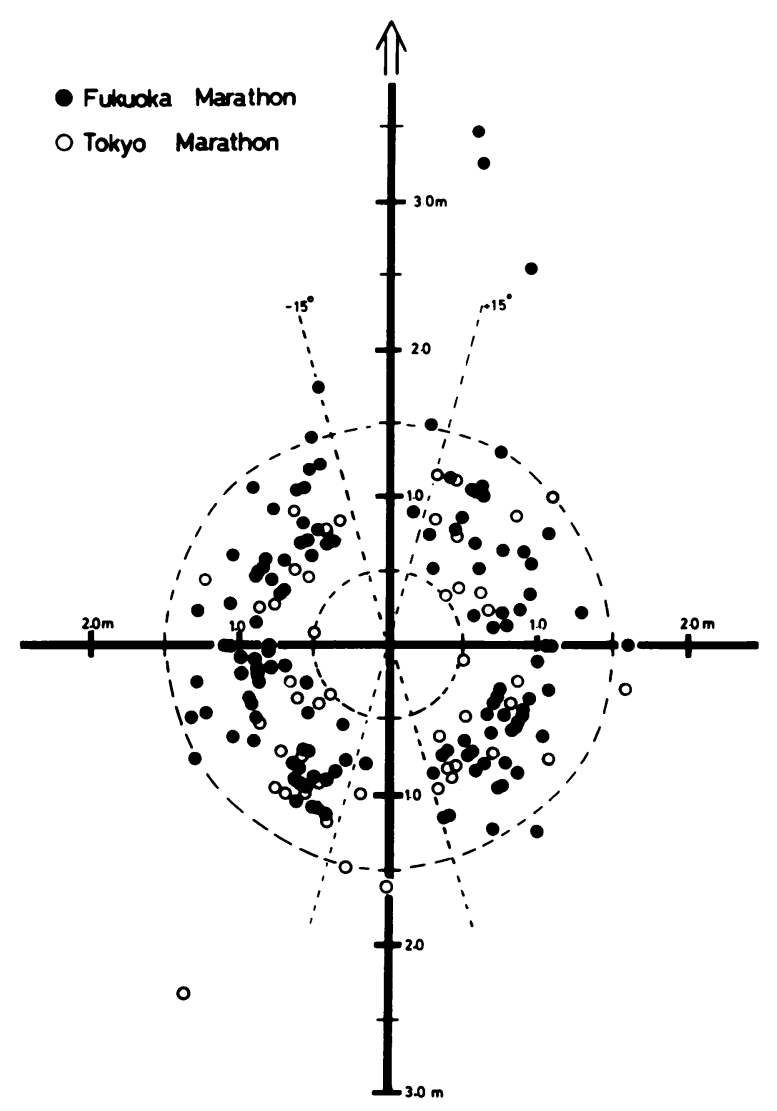

Fig. 4: The shortest inter-personal distance and the corresponding angular separation of the nearest rival relative to the direction of running. Points represent location of individual relative to direction of race (shown by arrow) and location of rival (assumed at intersection of horizontal and vertical axes).

running and (4) mutual supervision and surveillance over the course of the event. Against these potential benefits must be weighed possible disadvantages, including (1) a disturbance of personal running rhythms, (2) conformity with what may be an unsuitable running pace for the individual and (3) a possible decrease of mechanical efficiency due to (1) and (2).

It is particularly interesting that clustering is most marked among the elite performers. This may reflect both the greater importance of reducing wind resistance at higher speeds (resistance varies as the cube of velocity, Margaria, 1976; Pugh, 1976; Shephard, 1982) and the greater competitive stimulus in a fast moving group. Factors (3) and (4), on the other hand, probably assume greater importance for those participants who are at a lower level of competition.

The phenomenon of clustering among long-distance runners does not appear to have been noted previously but merits further study by those interested in marathon performance.

\section{References}

Margaria, R., 1976. Biomechanics and Energetics of Muscular Exercise. Clarendon Press, Oxford.

Pugh, L. G. C. E., 1976 "Air Resistance in Sport" pp. 149-164. In: Advances in Exercise Physiology. Eds.: E. Jokl, R. L. Anand and H. Stoboy. Karger, Basel.

Shephard, R. J., 1982. Physiology and Biochemistry of Exercise. Praeger, New York. 